AN. MED. INTERNA (Madrid) Vol. 18, N. ${ }^{\circ} 12$, pp. 641-643, 2001

\title{
Sarcoidosis pulmonar después de la instilación endovesical de mitomicina $\mathrm{C}$
}

\author{
M. A. CUERVO PINNA, C. CUERVO PINNA*, S. MACÍAS CASTILLO, J. C. BUREO \\ DACAL, $\mathrm{M}^{\mathrm{a}} \mathrm{J}$. ESPADA ALONSO**, J. PIMENTEL LEO***
}

Servicio de Medicina Interna. *Urología. **Centro de Salud La Paz. **Farmacología. ***Anatomía Patológica. Hospital Regional Universitario Infanta Cristina. Badajoz

\author{
LUNG SARCOIDOSIS FOLLOWING INSTILLATION OF MITOMYCIN \\ C IN THE URINARY BLADDER
}

\begin{abstract}
RESUMEN
La profilaxis intravesical con mitomicinaC (MMC) para la prevención de recurrencias del cáncer de vejiga, ha demostrado ser un tratamiento efectivo con pocos efectos secundarios. Sólo dos casos de toxicidad pulmonar después de la instilación endovesical de MMC han sido descritos en la literatura. Presentamos un paciente de 65 años que desarrolló una sarcoidosis pulmonar tras la administración intravesical de MMC. Esta asociación no había sido descrito hasta el momento actual. Asímismo se discuten las características clínicas y la patogenia de esta enfermedad pulmonar.
\end{abstract}

PALABRAS CLAVE:Sarcoidosis pulmonar. Mitomicina C endovesical.

\begin{abstract}
Intravesical prophiylaxis against recurrence of urinary bladder car cinomas using mitomycin C (MMC)has proved to be and effective treat ment with few side effects. Previously only two cases of lung toxicity after instillation of MMC into the urinary bladder has been described. We report a 65-year-old man in whom lung sarcoidosis occurred after intravesical administration of MMC. This association has not been reported to date. The clinical picture and the pathogenesis of this lung disease are discussed.
\end{abstract}

KEY WORDS: Lung sarcoidosis. Instillation of mitomycin $C$ in the uri nary bladder.

Cuervo Pinna MA, Cuervo Pinna C, Macías Castillo S, Bureo Dacal JC, Espada Alonso Má J, Pimentel Leo J. Sarcoidosis pulmonar después de la instilación endovesical de mitomicina C. An Med Interna (Madrid) 2001; 18: 641-643.

\section{INTRODUCCIÓN}

La sarcoidosis es una enfermedad sistémica de etiología desconocida que se caracteriza por la presencia de granulomas no necrotizantes. Se han implicado una gran variedad de agentes causales sin demostrar que ninguno de ellos sea un claro responsable.Sin embargo todos los datos apoyan el concepto de que se trata de una respuesta inmunitaria excesiva (adquirida, hereditaria o ambas) a un número limitado de antígenos o autoantígenos (1). La quimioterapia intravesical con mitomicina C (MMC) es utilizada en el tratamiento del cáncer superficial de vejiga por su conocido efecto de disminución de las recurrencias tumorales (2). Si bien la toxicidad pulmonar de la mitomicina por vía sistémica es un efecto secundario infrecuente pero bien descrito en la literatura $(3,4)$, la patología intersticial pulmonar en relación con la instilación vesical de MMC es excepcional habiéndose comunicado en sólo dos ocasiones $(5,6)$. Presentamos una asociación entre sarcoidosis pulmonar y el inicio terapéutico de MMC endovesical en un paciente diagnosticado de carcinoma papilar transicional de vejiga estadio A, lo cual no habiá sido previamente descrito (Base MEDLINE); posteriormente abordamos las hipótesis etiopatogénicas de esta enigmática enfermedad.

\section{CASO APORTADO}

Paciente de 65 años de edad remitido al servicio de Medicina Interna de nuestro hospital por disnea de dos meses de evolución y tos seca, que no mejoran a pesar de añadir al tratamiento broncodilatador previo (bromuro de ipratropio) terbutalina inhalada, teofilinas de liberación sostenida y ciclo de glucocorticoides orales $(1 \mathrm{mg} / \mathrm{kg} / \mathrm{d})$ las dos últimas semanas. Entre sus antecedentes personales destacaban: Enfermedad pulmonar obstructiva crónica (EPOC) controlada con anticolinérgicos inhalados, hábito tabáquico (10-15 cig/d), DM tipo 2 en tratamiento con glipicida y ser diagnosticado tres meses antes de carcinoma papilar transicional de vejiga grado I, estadio A (en radiografía de tórax realizada, entonces, para su estadiaje sólo se objetivó un atrapamiento aéreo bilateral). El paciente es sometido a resección transuretral y dos semanas después comienza con protocolo de instilación endovesical de mitomicina C (30 mg semanalmente el primer mes, seguido de una dosis de mantenimiento mensual de $30 \mathrm{mg}$ ) En el estudio epidemio-

Trabajo aceptado: 29 de Diciembre de 1999

Correspondencia: Miguel Angel Cuervo Pinna. Avda. Damián Téllez Lafuente, 1- 4․ 06010 Badajoz. E-mail: cuervo@grn.es 
lógico no se encontró una historia de exposición ambiental u ocupacional a berilio ni a productos de origen orgánico que pudiera inhalar. En la exploración física actual destacaba una respiración taquipneica (28 rpm) y una auscultación pulmonar donde se evidenciaban crepitantes finos dispersos por ambos campos pulmonares e hipoventilación en el hemitórax izquierdo; no se observaron adenopatías periféricas ni alteraciones cutáneas. Se practicó hemograma, coagulación, bioquímica general, proteinograma, determinación de anticuerpos antinucleares, ANCAs, anticuerpos antimitocondriales, analisis serológicos(aglutinaciones y Coombs a brucella, clamidia, VDRL, leismania, toxoplasma) ECG y ecografía abdominal, siendo los resultados normales. La gasometría arterial basal reveló una insuficiencia respiratoria parcial. La espirometría puso de manifiesto un transtorno ventilatorio mixto de predominio restrictivo. La determinación sérica de enzima convertidora de angiotensina (ECA) fue de $120 \mathrm{U} / 1(\mathrm{~N}<80)$. La radiografía de tórax demostró un infiltrado alveolointersticial bilateral de predominio en campo pulmonar izquierdo (Fig. 1), hallazgo observado en la TAC torácica, donde además se objetivaron adenomegalias de tamaño significativo en las diferentes cadenas ganglionares mediastínicas (Fig. 2). Se realizó entonces broncofibroscopia junto con broncoaspirado (BAS), lavado broncoalveolar (BAL) y biopsia transbronquial; el estudio citólogico, las baciloscopias y el cultivo en medio de Lowenstein del BAS no revelaron atipias ni presencia de micobacterias. En el BAL se encontró un $34 \%$ de células con características citométricas linfoides; aplicando una batería de anticuerpos monoclonales se obtuvieron los resultados que se reflejan en la tabla I. En el estudio anatomopatológico se observaban abundantes formaciones granulomatosas con histiocitos, células epitelioides y células gigantes multinucleadas, todo ello compatible con una inflamación granulomatosa no necrotizante. Con el diagnóstico de sarcoidosis pulmonar estadio III, se procedió a la retirada del quimioterápico y al mantenimiento de los glucocorticoides orales (prednisona $40 \mathrm{mg} /$ día). Dos semanas después de suspender la terapéutica citostática endovesical el paciente presentó una mejoría progresiva de la sintomatología respiratoria A los 3 meses el enfermo se encontraba asintomático, el estudio espirométrico mostró un patrón obstructivo leve y el infiltrado alvelointersticial asícomo las adenopatías mediastínicas habían prácticamente desaparecido (control por TAC). A partir de entonces se inició la reducción progresiva de esteroides, suprimiéndolos a los 6 meses, sin evidencia en la actualidad de recidiva de la enfermedad pulmonar (6 meses tras finalizar el tratamiento).

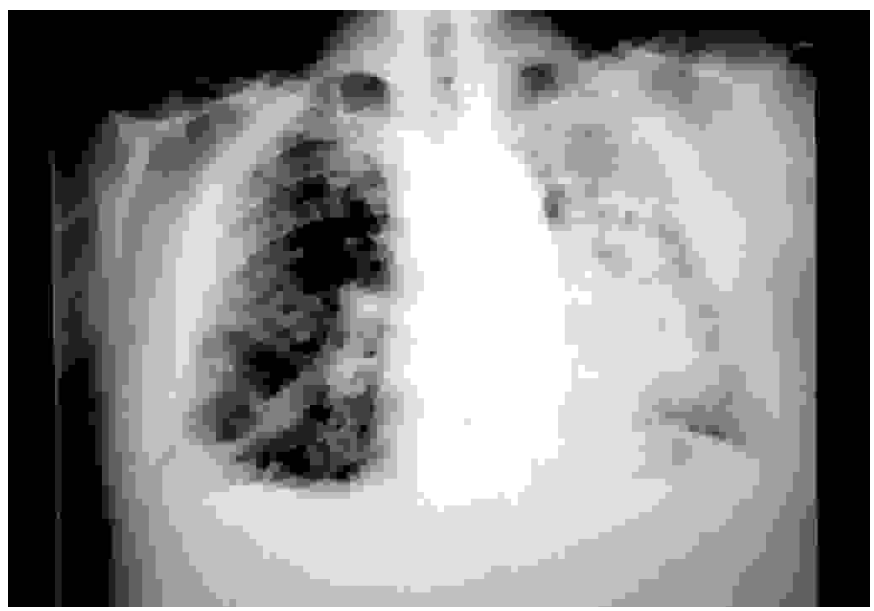

Fig. 1. Inflitrado alveolointersticial bilateral de predominio en campo pulmonar izquierdo.

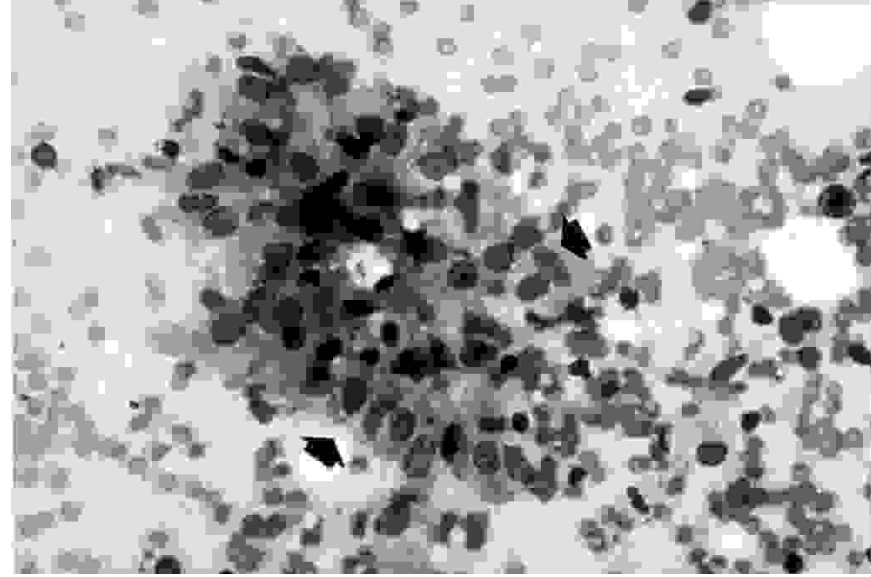

Fig. 2. Acúmulo de células epitelioides, con al gunos núcleos de forma alargada, sin existencia de necrosis (M GG*, x 200); *: M ayGrünwald-Giemsa.

TABLA I

PO BLACIÓ N LINFOIDE EN EL BAL (\%)

\begin{tabular}{llll} 
CD 45 & 93,3 & CD 19 & 0,2 \\
CD 2 & 92 & CD 56 & 9,7 \\
CD 3 & 87,7 & DR & 22,8 \\
CD 4 & 73 & CD 25 & 5 \\
CD 8 & 14,2 & CD 38 & 35,6 \\
TCR $\alpha \beta$ & 90 & CD 45 RA & 3 \\
TCR $\delta / \gamma$ & 1,2 & CD 45 RO & 83,5 \\
CD 14 & 0,2 & Cociente CD 4/CD 8 & 5,14 \\
CD 16 & 1,5 & & \\
\hline
\end{tabular}

DISCUSIÓN

La etiología de la sarcoidosis sigue siendo desconocida por una serie de razones que incluyen la heterogenicidad de sus manifestaciones clínicas, la falta de una definición precisa (7), la superposición clínica con otras enfermedades, y la poca sensibilidad y especifidad de los test diagnósticos de los que disponemos (8). Se han descrito casos de formas familiares de sarcoidosis (9) así como en colectivos de enfermeras (10), lo que sugiere una transmisión de persona a persona o la exposición a un agente ambiental.La inhalación de ciertos metales, en especial el berilio (11) y agentes orgánicos dan lugar a enfermedades que son histológicamente y clínicamente indistinguibles de la sarcoidosis, lo que apoyaría la hipótesis ambiental. Recientemente se ha comunicado la sociación esta interferon alfa (en pacientes con Hepatitis C) y la aparición de sarcoidosis pulmonar (12). Múltiples microorganismos inducen inflamación granulomatosa, de los cuales las micobacterias han sido las más estudiadas (13). La sarcoidosis ocurre con más frecuencia en gemelos monocigotos que en dicigotos por lo que los factores genéticos parecen jugar un papel importante en su patogénesis. En la búsqueda de genes que puedan conferir susceptibilidad a padecer sarcoidosis, múlti- 
ples estudios serológicos han identificado asociaciones con antígenos de clase I (HLA-A1 y B8) y de clase II (HLA-DR3) (14), si bien estos resultados son similares a los encontrados en otras enfermedades autoinmunes y la fuerza de la asociación es menor en la sarcoidosis.

En el caso que presentamos, una vez descartadas razonablemente todas las causas conocidas de enfermedad granulomatosa pulmonar, procedimos a la realización de un BAL y de biopsia transbronquial. En el BAL (Tabla I) se observó una población linfocitaria formada mayoritariamente por células $\mathrm{T}$ (CD2, CD3) con una distribución porcentual entre CD4 y CD8 elevada, con signos de activación celular (DR, CD25, CD38), hallazgos que aunque característicos de la sarcoidosis, en modo alguno son específicos de esta enfermedad (15). El estudio anatomopatológico reveló la presencia de granulomas no necrotizantes condición indispensable pero tampoco suficiente para el diagnóstico de sarcoidosis. Así pues ningún dato hematológico, radiológico, inmunológico ni histológico es específico para esta entidad y permanece como un diagnóstico de exclusión en ausencia de un agente causal conocido. El paciente había sido diagnosticado tres meses antes de carcinoma transicional de vejiga, estadio A de Jewett (invasión de la submucosa), y estaba siendo sometido a un régimen terapéutico de MMC endovesical (un total de $170 \mathrm{mg}$ hasta su supensión). La MMC es un agente quimioterápico antibiótico que actúa a través de la inhibición de la síntesis de DNA. Tiene un peso molecular más alto que el resto de citostáticos empleados en terapéutica intravesical adyuvante (334 dalton), y en consecuencia se han descrito menos problemas relacionados con su absorción a través del urotelio (se absorbe menos del 1\% de la MMC instilada) (16). Parece más efectiva que otros agentes quimioterapéuticos endovesicales en el tratamiento primario de cánceres vesicales en el estadio A (17). Los efectos colaterales de la MMC endovesical incluyen cistitis química (10 a 15\%) y dermatitis de contacto (18). Asímismo han sido documentadas reacciones alérgicas

\section{Bibliografía}

1. Arribas Castrillo JM. Sarcoidosis. An Med Interna (Madrid) 2000; 17: 513-516.

2. Tolley DA, Hargreave TB, Smith PH, Williams JL, Grigor KM, Parmar $\mathrm{MK}$, et al. Effective intravesical mitomycin $\mathrm{C}$ on recurrence of newly diagnosed superficial bladder cancer; a report from the medical research council subgroup on superficial bladder. BMJ 1988; 296: 1759-1761.

3. Okuno SH, Frytak S. Mitomycin lung toxicity. Acute and chronic phases. Am J Clin Oncol 1997; 20: 282-4.

4. Castro M, Veeder MH, Mailliard JA, Tazelaar HD, Jett JR. A prospecti ve study of pulmonary function in patients receiving mitomycin. Chest 1996; 109: 939-944.

5. Neubauer H, Blech M, Wilhelms E, Criee CP, Neuhaus KL, Truss F Pulmonary fibrosis following instillation of mitomycin $\mathrm{C}$ in the urinary bladder. Urologe 1987; 26: 41-3.

6. Wilhelms E, Criee CP, Neubauer H, Neuhaus KL. Lung parenchyma disease following instillation of mitomycinC in the bladder. Dtsch Med Wochenscher 1986; 111: 1564-6.

7. Yamamoto M, Sharma OP, Hosado Y. The 1991 descriptive definition of sarcoidosis. Sarcoidosis 1992; 9: Suppl 1: 33-4.

8. Lee S. Newman, Cecile S. Rose, Lisa A. Maier. Sarcoidosis (Review Article). N Engl J Med 1997; 336: 1224-1334.

9. Harrington DW, Major M, Rybicki B, Popovich J, Maliarik M, Lannuzzi MC. Familial Sarcoidosis: analysis of 91 families. Sarcoidosis 1994; 11: 240-3.

10. Endmonstone W.M. Sarcoidosis in nurses: is there an association? Thorax 1988; 43: 342-3.

11. Newman L.S. Beryllium disease and sarcoidosis: clinical and laboratory
(19) y calcificación de la vejiga (20). Si bien la toxicidad pulmonar de la mitomicina por vía sistémica es un efecto secundario infrecuente pero descrito en la literatura (2), la patología intersticial pulmonar asociada a la instilación vesical de mitomicina es excepcional, habiéndose comunicado sólo 2 casos (4, 5). En el caso que se presenta hasta que no se procedió a la supresión de la MMC no se inició la resolución de la patología granulomatosa pulmonar, con lo cual barajamos la posibilidad de que pudiera existir una relación causal. Fueron Wilhelms et al (6) quienes comunicaron por primera vez en 1986 un caso de enfermedad parenquimatosa pulmonar tras la administración intravesical de MMC, que se resolvió con la suspensión de las instilaciones y el tratamiento corticoideo. Al año siguiente Neubauer y el grupo de Wilhelms (5) publicaron el hallazgo de una fibrosis pulmonar tras la instilación de este quimioterápico. La sarcoidosis pulmonar tras la administración intravesical de MMC no ha sido descrita hasta el momento actual (revisión realizada a través de la base MEDLINE). Probablemente la relación causal entre la terapia intravesical con MMC y sarcoidosis pulmonar, en nuestro caso, hubiera quedado demostrada si tras reintroducir las instilaciones endovesicales apareciera nuevamente la patología granulomatosa pulmonar; ello no nos parecía ético dadas las restantes opciones terapéuticas igualmente válidas de las que disponemos en la actualidad. Así pues, la hipótesis de que un antibiótico como la MMC se pudiera comportar como un antígeno exógeno que desencadenara una respueste inmune exagerada y conducir a la formación de granulomas en un paciente genéticamente predispuesto, ampliaría un poco más el espectro etiopatogénico de esta enigmática enfermedad. En el momento de la fecha, un estudio multicéntrico está siendo conducido por el "National Heart, Lung, and Blood Institute" para abordar las hipótesis ambientales, infecciosas, genéticas e inmunológicas sobre la patogénesis de la sarcoidosis, y conoceremos si agentes exógenos como la mitomicina están siendo involucrados. links. Sarcoidosis 1995; 12: 7-19.

12. Vallina E, García Díez A, Gallego M, Villaverde P, Rippe ML, Arribas JM. A Propósito de un sarcoidosis pulmonar inducido por tratamiento con interferón alfa en una paciente con hepatitis C. An Med Interna (Madrid) 2000; 17: 538-539.

13. Mangiapan G, Hance AJ. Mycobacteria and sarcoidosis: and overview and summary of recent molecular biological data. Sarcoidosis 1995; 12: 20-37.

14. Pasturenzi L, Martinetti M, Cuccia M, Cipriani A, Semenzato G, Lisetti M. HLA class I, II and III polymorphism in Italian patients with sarcoidosis; the Pavia-Padova Sarcoidosis Study Group. Chest 1993; 104: 1170-5.

15. Winterbauer RH, Lammert J, Selland M, Wu R, Corley D, Springmeyer SC. Bronchoalveolar lavage cell populations in the diagnosis of sarcoidosis. Chest 1993; 104: 352-61

16. Van Helsdingen PJ, Rikken CH, Sleeboom HP. Mitomycin C resorption following repeated intravesical instillations using different instillations times. Urol. Int 1988; 43-42.

17. Heney NM, Koontz WW, Barton B. Intravesical thiotepa versus mitomycinC in patients with TA, T1 and TIS transitional cell carcinoma of the bladder: A phase III prospective randomized study. J. Urol. 1988;140: 390.

18. William J, Catalona.Tumores uroteliales del tracto urinario. En: Campbell Urología.Madrid.Editorial Médica Paramericana, 1994;1090-1154.

19. Trasher JB, Crawford ED. Complications of intravesical chemotherapy. Urol Clin North Am 1992; 19: 529-39.

20. Mejias GJ, Pelluch AA, Romero PP, Fernández GJ, Gasso MM, Sánchez MM, et al.Calcified bladder lesions secondary to the instillation of mitomycin C. Arch Esp Urol 1991; 44: 1057-60. 